\title{
On multiplicative independence of rational function iterates
}

\author{
Marley Young ${ }^{1}$
}

Received: 23 September 2017 / Accepted: 15 February 2020 / Published online: 21 February 2020

(c) The Author(s) 2020

\begin{abstract}
We give lower bounds for the degree of multiplicative combinations of iterates of rational functions (with certain exceptions) over a general field, establishing the multiplicative independence of said iterates. This leads to a generalisation of Gao's method for constructing elements in the finite field $\mathbb{F}_{q^{n}}$ whose orders are larger than any polynomial in $n$ when $n$ becomes large. Additionally, we discuss the finiteness of polynomials which translate a given finite set of polynomials to become multiplicatively dependent.
\end{abstract}

Keywords Iteration $\cdot$ Multiplicative dependence $\cdot$ Rational function

Mathematics Subject Classification 11R18 · 39B12 · 12E99 · 37F10

\section{Introduction and main results}

We say that $n$ non-zero elements $a_{1}, \ldots, a_{n}$ of a ring are multiplicatively independent if, for integers $k_{1}, \ldots, k_{n}$, we have that $a_{1}^{k_{1}} \ldots a_{n}^{k_{n}}=1$ if and only if $k_{1}=\cdots=k_{n}=0$. Otherwise we say they are multiplicatively dependent. Multiplicative independence, especially of values of polynomials and rational functions, is being increasingly studied. In [4], Bombieri, Masser and Zannier initiate study of the intersection of algebraic curves with proper algebraic subgroups of the multiplicative group $\mathbb{G}_{m}^{n}$. It turns out (see [3, Corollary 3.2.15]) that each such subgroup of $\mathbb{G}_{m}^{n}$ is defined by finitely many equations of the form $X_{1}^{k_{1}} \ldots X_{n}^{k_{n}}=1$, where $k_{1}, \ldots, k_{n}$ are integers, not all zero. As such, [4], which leads into the area of "unlikely intersections", really concerns the multiplicative dependence of points on curves.

Communicated by Umberto Zannier.

$凶 \quad$ Marley Young

mjy28@cam.ac.uk

1 Department of Pure Mathematics and Mathematical Statistics, University of Cambridge, Cambridge CB3 0WB, UK 
More recently, we see multiplicative independence being studied in the context of arithmetic dynamics. In [18], it is shown that under fairly natural conditions on rational functions $f_{1}, \ldots, f_{s}$ over a number field $\mathbb{K}$, the values $f_{1}(\alpha), \ldots, f_{s}(\alpha)$ are multiplicatively independent for all but finitely many $\alpha \in \mathbb{K}^{\mathrm{ab}}$, where $\mathbb{K}^{\mathrm{ab}}$ is the maximal abelian extension of $\mathbb{K}$. This leads to results on multiplicative dependence in the orbits of a univariate polynomial dynamical system.

Clearly, to study the multiplicative independence of elements in the orbits of polynomials or rational functions, it is necessary to know when the given functions are multiplicatively dependent, as in this case all their values must be multiplicatively dependent. We study this problem in the context of iterates of rational functions over a field.

Throughout the paper, $\mathbb{F}$ will denote a field of characteristic $p$ (zero or prime), and $f \in \mathbb{F}(X)$ a non-constant rational function in lowest terms over $\mathbb{F}$. That is, $f=g / h$ with $d:=\operatorname{deg} f=\max \{\operatorname{deg} g, \operatorname{deg} h\} \geq 1$. Being in "lowest terms" means $\operatorname{gcd}(g, h)=1$, or equivalently, $g$ and $h$ share no roots in any extension field of $\mathbb{F}$. As such, when referring to zeros and poles of a rational function, we mean roots of its numerator and denominator respectively in an algebraic closure $\overline{\mathbb{F}}$ of $\mathbb{F}$. We recursively define the iterates of $f$ by

$$
f^{(0)}(X)=X, \quad \text { and } \quad f^{(k)}=f \circ f^{(k-1)} \text { for } k \geq 1 .
$$

In [10], Gao considers the multiplicative independence of polynomials over a finite field $\mathbb{F}_{q}$, where $q$ is a prime power, proving that if $f \in \mathbb{F}_{q}[X]$ is not a monomial or certain binomial, then the iterates $f^{(1)}, \ldots, f^{(n)}$ are multiplicatively independent for $n \geq 1$. Gao uses this fact to give a method for constructing elements of "high order" in $\mathbb{F}_{q^{n}}$ when $q$ is fixed. That is, elements with order larger than any polynomial in $n$ when $n$ is large. In particular, if we define $\bar{n}=q^{\left\lceil\log _{q} n\right\rceil}$, and $g \in \mathbb{F}_{q}[X]$ is not a monomial or certain binomial, then any root of an irreducible factor of degree $n$ of $X^{\bar{n}}-g(X)$ is an element in $\mathbb{F}_{q^{n}}$ of order at least

$$
n^{\frac{\log _{q} n}{4 \log _{q}\left(2 \log _{q} n\right)}-\frac{1}{2}}
$$

Sharper analysis of the same method by Popovych in [19] improves the lower bound on the order to

$$
\left(\begin{array}{c}
n+t-1 \\
t
\end{array}\right) \prod_{i=0}^{t-1} \frac{1}{d^{i}}
$$

where $d=\left\lceil 2 \log _{q} n\right\rceil$ and $t=\left\lfloor\log _{d} n\right\rfloor$.

In the case of rational functions over a general field, we also have multiplicative independence of iterates, up to a few exceptional cases. We remark (see Lemma 2.8) that these exceptions are precisely the rational functions which, under iteration, eventually become a monomial. For example, if $f^{(n)}(X)=X^{k}$, then $f^{(n)}(X)$ and $f^{(2 n)}(X)=X^{k^{2}}$ are multiplicatively dependent. Note also that the cases of zero and 
positive characteristic are different. One distinction, of course, is the existence of inseparable maps in fields of positive characteristic. We see in Lemma 2.7, that this corresponds to a difference in which rational functions have an iterate which is a polynomial, let alone a monomial. Moreover, especially in the polynomial case, positive characteristic allows terms in iterates to vanish which would otherwise prevent them from becoming monomials.

Theorem 1.1 Suppose that $f=g / h \in \mathbb{F}(X)$ has degree $d \geq 2$, and is not a monomial of the form $a X^{ \pm d}$, nor of the form $L\left(X^{p^{\ell}}\right)$, where $L \in \mathbb{F}(X)$ has degree 1 and $\ell$ is a positive integer. Let $n \geq 1$, and write

$$
\Psi(n)=\min _{\substack{k_{1}, \ldots, k_{n} \in \mathbb{Z} \\ k_{n} \neq 0}}\left(\operatorname{deg}\left(\left(f^{(1)}\right)^{k_{1}} \ldots\left(f^{(n)}\right)^{k_{n}}\right)\right) .
$$

Then there exists an integer $j \geq 0$ depending only on $f$ such that $\Psi(n) \geq d^{n}$ if $n \leq j$, and $\Psi(n) \geq d^{n-j}$ if $n>j$.

It is easy to show that the above result implies the multiplicative independence of iterates of $f$.

Corollary 1.2 Suppose that $f=g / h \in \mathbb{F}(X)$ has degree $d \geq 2$, and is not of the form $a X^{ \pm d}$, or $L\left(X^{p^{\ell}}\right)$, where $L \in \mathbb{F}(X)$ has degree 1 and $\ell$ is a positive integer. Then for any integer $n \geq 1$, the iterates $f^{(1)}, \ldots, f^{(n)}$ are multiplicatively independent, even up to constants.

Proof If $\left(f^{(1)}\right)^{k_{1}} \ldots\left(f^{(n)}\right)^{k_{n}}=c, c \in \mathbb{F}$, then Theorem 1.1 ensures $k_{n}=0$, as otherwise the degree would be positive. Then we get $k_{n-1}=\cdots=k_{1}=0$ recursively.

In the polynomial case, we also obtain a lower bound on the number of distinct zeros of a multiplicative combination of iterates.

Theorem 1.3 Suppose $f \in \mathbb{F}[X]$ has degree $d \geq 2$, and has non-vanishing derivative. Let $\mathrm{z}(f)$ denote the number of distinct zeros of $f$ (in an algebraic closure of $\mathbb{F}$ ), and for an integer $n$ define

$$
Z(n):=\min _{\substack{k_{1}, \ldots, k_{n} \in \mathbb{Z} \\ k_{n} \neq 0}}\left(\mathrm{z}\left(\left(f^{(1)}\right)^{k_{1}} \ldots\left(f^{(n)}\right)^{k_{n}}\right)\right) .
$$

Let $e$ be the least positive integer $k$ such that $f^{(k)}(0)=0$, and say that $e=\infty$ if $f^{(k)}(0) \neq 0$ for all $k \geq 1$. Suppose that $f(0) \neq 0$ and $\mathrm{z}(f)>1$, or that $\mathrm{z}(f)>2$. Then $Z(n) \geq \gamma(f) d^{n-1}+1$ if $n \leq e$, and $Z(n) \geq d^{n-e}+1$ when $n>e$, where

$$
\gamma(f)= \begin{cases}\max \{\mathrm{z}(f)-2,1\}, & \text { if } \mathbb{F} \text { has characteristic } 0 \\ 1, & \text { otherwise. }\end{cases}
$$

We use Corollary 1.2 in the following extension of the main theorem in [10]. 
Theorem 1.4 Let $q$ be a prime power and $n \geq 1$ an integer. Let $g, h \in \mathbb{F}_{q}[X]$ be coprime with $\operatorname{deg} h, \operatorname{deg} g \leq d=\left\lceil 2 \log _{q} n\right\rceil$, and suppose $f=g / h$ satisfies the conditions from Corollary 1.2. Suppose that $\alpha \in \mathbb{F}_{q^{n}}$ has degree $n$ over $\mathbb{F}_{q}$ and is a root of $X^{m} h(X)-g(X)$, where $m=\bar{n}=q^{\left\lceil\log _{q} n\right\rceil}$. Then for

$$
s= \begin{cases}n-1, & f \in \mathbb{F}[X], \\ \lfloor(n-1) / 2\rfloor, & \text { otherwise, }\end{cases}
$$

and $t=\left\lfloor\log _{d} n\right\rfloor, \alpha$ has order in $\mathbb{F}_{q^{n}}$ at least

$$
\left(\begin{array}{c}
s+t \\
t
\end{array}\right) \prod_{i=0}^{t-1} \frac{1}{d^{i}}
$$

As an aside we additionally ask, given rational functions $F_{1}, \ldots, F_{n} \in \mathbb{F}(X, Y)$ and polynomial $u \in \mathbb{F}[X]$, when $F_{1}(X, u(X)), \ldots, F_{n}(X, u(X))$ are multiplicatively dependent. In particular, we find upper bounds on the degree of $u$ such that this possible, and the number of monic $u$ for which this is the case.

Theorem 1.5 Suppose $\mathbb{F}$ is a field of characteristic zero, $n$ is a positive integer, and $F_{i}=G_{i} / H_{i} \in \mathbb{F}(X, Y)$ are rational functions for $1 \leq i \leq n$, of respective degrees $d_{1} \leq \cdots \leq d_{n}$ in $X$ and $1 \leq e_{1} \leq \cdots \leq e_{n}$ in $Y$. For $1 \leq i \neq j \leq n$, define

$$
R_{i j}(X)=\operatorname{Res}_{Y}\left(G_{i}, G_{j}\right) \operatorname{Res}_{Y}\left(G_{i}, H_{j}\right) \operatorname{Res}_{Y}\left(H_{i}, G_{j}\right) \operatorname{Res}_{Y}\left(H_{i}, H_{j}\right)
$$

where $\operatorname{Res}_{Y}(P, Q)$ is the resultant of $P, Q \in \mathbb{F}[X, Y]$, considered as polynomials in $Y$, and set

$$
E=\sum_{1 \leq i<n} \sum_{i<j \leq n} \operatorname{deg} R_{i j}
$$

If $R_{i j} \not \equiv 0$ for all $i \neq j$, then there are finitely many monic polynomials $u \in \mathbb{F}[X]$ such that

$$
F_{1}(X, u(X)), \ldots, F_{n}(X, u(X))
$$

are multiplicatively dependent. In particular, such a $u$ has degree not exceeding $E+$ $2 d_{n}-1$.

Recalling that the resultant of two polynomials of respective degrees $m$ and $n$ is a polynomial in the coefficients of degree $m+n$, and that each $G_{i}, H_{i}$ written as a polynomial in $Y$, has degree at most $e_{n}$, with each coefficient having degree not exceeding $d_{n}$. We have that for $i \neq j, \operatorname{deg} \operatorname{Res}_{Y}\left(G_{i}, G_{j}\right) \leq\left(e_{n}+e_{n}\right) d_{n}=2 d_{n} e_{n}$, and the same bound holds for all the factors of the polynomial $R_{i j}$ defined above. Thus, counting $\frac{n(n-1)}{2}$ distinct pairs $\{i, j\}$, we obtain $E \leq 4 n(n-1) d_{n} e_{n}$. 
Theorem 1.5 can be applied to the particular scenario of shifting a given set of polynomials by a polynomial $u$, giving a analogue of results for algebraic numbers from [4] and [7].

Corollary 1.6 Suppose $\mathbb{F}$ has characteristic zero, $n$ is a positive integer and $f_{1}, \ldots, f_{n} \in$ $\mathbb{F}[X]$ are distinct polynomials, not all constant, of respective degrees $d_{1} \leq \cdots \leq d_{n}$ and let

$$
C=d_{n} \frac{n(n-1)}{2}
$$

Then there are at most $\left(\begin{array}{c}2 C+3 d_{n}-1 \\ C\end{array}\right)$ monic polynomials $u \in \mathbb{F}[X]$ such that

$$
f_{1}+u, \ldots, f_{n}+u
$$

are multiplicatively dependent. In particular, such a $u$ has degree not exceeding $C+$ $2 d_{n}-1$.

The paper is organised with sections corresponding to proofs of the main theorems: In the next section, we collect various results on iterates of rational functions, specifically concerning zeros and poles which are common to different iterates, and the degrees of the numerator and denominator of iterates. We use these results to bound from below the number (counted with multiplicity) of zeros and poles of a given iterate which cannot be found in any of the previous ones. We thus obtain Theorem 1.1. In Sect. 3, we give the proof of a version of [8, Main Theorem], which holds for polynomials over fields of arbitrary characteristic. This is used in conjunction with the general method from Sect. 2 to prove Theorem 1.3. In Sect. 4, we discuss elements of high order in finite fields in a manner analogous to [10,19], but in a slightly more general setting. Finally, in Sect. 5, we use resultants in conjunction with the polynomial $A B C$-theorem to prove Theorem 1.5.

\section{Proof of Theorem 1.1}

To prove Theorem 1.1, we need some facts about the composition of rational functions. Let $u=v / w, F=G / H \in \mathbb{F}(X)$ be in lowest terms over $\mathbb{F}$, and write

$$
u(X)=\frac{v(X)}{w(X)}=\frac{a_{l} X^{l}+\cdots+a_{s} X^{s}}{b_{m} X^{m}+\cdots+b_{t} X^{t}}, a_{l}, a_{s}, b_{m}, b_{t} \neq 0
$$

with $\operatorname{deg} u \geq 1$. Let $u \circ F=P / Q$. Recall that the degree of a rational function $f \in \mathbb{F}(X)$, written is lowest terms, is equal to the degree $[\mathbb{F}(X): \mathbb{F}(f(X))]$, and hence by the product formula for degrees of extensions,

$$
\operatorname{deg} u \circ F=(\operatorname{deg} u)(\operatorname{deg} F) .
$$


Next, we have

$$
\begin{aligned}
\frac{P(X)}{Q(X)} & =\frac{a_{l}\left(\frac{G(X)}{H(X)}\right)^{l}+\cdots+a_{s}\left(\frac{G(X)}{H(X)}\right)^{s}}{b_{m}\left(\frac{G(X)}{H(X)}\right)^{m}+\cdots+b_{t}\left(\frac{G(X)}{H(X)}\right)^{t}} \\
& =H(X)^{m-l} G(X)^{s-t} \frac{q(X)}{r(X)}
\end{aligned}
$$

where

$$
q(X)=\sum_{i=0}^{l-s} a_{l-i} G(X)^{l-s-i} H(X)^{i} \text { and } r(X)=\sum_{i=0}^{m-t} b_{m-i} G(X)^{m-t-i} H(X)^{i}
$$

Note that a composition of rational functions in lowest terms is itself in lowest terms ([6, Lemma 2.2] is easily extended to our situation). In particular, $G, H, q$ and $r$ are pairwise relatively prime. This means we need not worry about the possibility of factors cancelling after composition. Hence, from (4), whenever $\operatorname{deg} G \neq \operatorname{deg} H$ we have

$$
\begin{aligned}
& \operatorname{deg} P=\operatorname{deg} H(\operatorname{deg} u-l)+(\operatorname{deg} G) s+\operatorname{deg} F(l-s), \\
& \operatorname{deg} Q=\operatorname{deg} H(\operatorname{deg} u-m)+(\operatorname{deg} G) t+\operatorname{deg} F(m-t),
\end{aligned}
$$

where $P / Q$ is in lowest terms.

We can use these facts to obtain results about which zeros and poles are common to different iterates of $f$. It turns out that these relations depend primarily on the earliest iterates of $f$ to have either 0 or a point at infinity as a zero or pole. We hence set the following notation.

Definition 2.1 Write $f^{(k)}=g_{k} / h_{k}$ for the $k$-th iterate of $f$ in lowest terms, and let $e$ be defined as in Theorem 1.3. Further define $\epsilon, \mu$ and $v$ to be respectively the smallest positive integers $k$ such that $h_{k}(0)=0, \operatorname{deg} g_{k}<\operatorname{deg} h_{k}$, and $\operatorname{deg} g_{k}>\operatorname{deg} h_{k}$. These again take the value $\infty$ if their respective conditions are not satisfied for any $k \geq 1$.

We first note that there are restrictions on the possible combinations of the values $e, \epsilon, \mu, \nu$. We make particular use of the next result.

Lemma 2.2 Suppose $\mu<v$ and $\epsilon<\infty$. Then $\mu \leq \epsilon<e<\infty$, and in particular $e=\epsilon+\mu$.

Proof If $\epsilon<\mu$, then by definition we have $\operatorname{deg} g_{\epsilon}=\operatorname{deg} h_{\epsilon}=\operatorname{deg} f^{(\epsilon)}=d^{\epsilon}$ and $\operatorname{deg} g_{\mu-\epsilon}=\operatorname{deg} h_{\mu-\epsilon}=\operatorname{deg} f^{(\mu-\epsilon)}=d^{\mu-\epsilon}$. Hence, upon setting $u=f^{(\epsilon)}$ and $F=f^{(\mu-\epsilon)},(5)$ gives

$$
\begin{aligned}
\operatorname{deg} g_{\mu} & =\operatorname{deg} h_{\mu-\epsilon}\left(\operatorname{deg} f^{(\epsilon)}-\operatorname{deg} g_{\epsilon}\right)+\operatorname{deg} g_{\mu-\epsilon} s+\operatorname{deg} f^{(\mu-\epsilon)}\left(\operatorname{deg} g_{\epsilon}-s\right) \\
& =d^{\mu-\epsilon}\left(d^{\epsilon}-d^{\epsilon}\right)+d^{\mu-\epsilon} s+d^{\mu-\epsilon}\left(d^{\epsilon}-s\right)=d^{\mu} \geq \operatorname{deg} h_{\mu} .
\end{aligned}
$$

This contradicts the definition of $\mu$, and so we must have $\mu \leq \epsilon$. 
Furthermore, if $e<\epsilon$, then $f^{(\epsilon-e)}(0)=f^{(\epsilon-e)}\left(f^{(e)}(0)\right)=f^{(\epsilon)}(0)$, so 0 is a pole of $f^{(\epsilon-e)}$, contradicting the choice of $\epsilon$. Hence we have $\epsilon<e$, and by setting $u=f^{(j)}, F=f^{(\epsilon)}$ for a positive integer $j$, (4) gives that 0 is a zero of $f^{(\epsilon+j)}$ if and only if $\operatorname{deg} g_{j}<\operatorname{deg} h_{j}$. Thus $e=\epsilon+\mu$.

We have the following extension of a result of Gao [10, Lemma 2.2].

Lemma 2.3 For all integers $k>\ell \geq 1$,

(i) A zero of $f^{(\ell)}$ is a zero of $f^{(k)}$ if and only if $e<\infty$ and $k \equiv \ell(\bmod e)$.

(ii) A pole of $f^{(\ell)}$ is a pole of $f^{(k)}$ if and only if $\operatorname{deg} g_{k-\ell}>\operatorname{deg} h_{k-\ell}$.

(iii) A pole of $f^{(\ell)}$ is a zero of $f^{(k)}$ if and only if $\operatorname{deg} g_{k-\ell}<\operatorname{deg} h_{k-\ell}$.

(iv) If $\mu<v$, then a zero of $f^{(\ell)}$ is a pole of $f^{(k)}$ if and only if $\epsilon<e<\infty$ and $k \equiv \ell-\mu(\bmod e)$.

Proof Let $k>\ell \geq 1$. For part (i), suppose that a zero $\alpha$ of $f^{(\ell)}$ is a zero of $f^{(k)}$. Then $f^{(k)}(\alpha)=f^{(\ell)}(\alpha)=0$. As $f^{(k)}=f^{(k-\ell)} \circ f^{(\ell)}$, we have

$$
f^{(k-\ell)}(0)=f^{(k-\ell)}\left(f^{(\ell)}(\alpha)\right)=f^{(k)}(\alpha)=0
$$

Thus we must have $e<\infty$, so assume this is the case. If $k \equiv \ell(\bmod e)$, say $k=\ell+j e$ where $j \geq 1$, then for any zero $\beta$ of $f^{(\ell)}$,

$$
f^{(k)}(\beta)=f^{(j e)}\left(f^{(\ell)}(\beta)\right)=f^{(j e)}(0)=0 .
$$

Hence any zero of $f^{(\ell)}$ is a zero of $f^{(k)}$. Now, suppose $k \neq \equiv \ell(\bmod e)$, say $k=$ $\ell+j e+r$ where $u \geq 0$ and $1 \leq r<e$. If $f^{(k)}$ and $f^{(\ell)}$ have a zero in common then, by the above argument, $f^{(j e+r)}(0)=f^{(k-\ell)}(0)=0$. But then

$$
f^{(r)}(0)=f^{(r)}\left(f^{(j e)}(0)\right)=f^{(j e+r)}(0)=0,
$$

contradicting the choice of $e$. Therefore $f^{(k)}$ and $f^{(\ell)}$ have no zero in common when $k \not \equiv \ell(\bmod e)$.

Writing $f^{(k)}=f^{(k-\ell)} \circ f^{(\ell)}$, the second and third parts follow immediately from (4).

Now, suppose that $\mu<v$. By definition, we have that deg $g_{k}=\operatorname{deg} h_{k}$ for $1 \leq k<$ $\mu$. Set $u=f^{(j)}, F=f^{(\mu)}$, so $f^{(\mu+j)}=u \circ F=P / Q$ as in (4). If $e, \epsilon>j \geq 1$, then in (5) and (6), $s=t=0$ and so $\operatorname{deg} g_{\mu+j}=\operatorname{deg} h_{\mu+j}=d^{\mu+j}$. We thus note that

$$
\operatorname{deg} g_{k}=\operatorname{deg} h_{k}=d^{k} \quad \text { for all } 1 \leq k \neq \mu<\mu+\min \{\epsilon, e\} .
$$

Suppose a zero $\alpha$ of $f^{(\ell)}$ is a pole of $f^{(k)}$. Then we have

$$
f^{(k-\ell)}(0)=f^{(k-\ell)}\left(f^{(\ell)}(\alpha)\right)=f^{(k)}(\alpha),
$$


and so 0 is a pole of $f^{(k-\ell)}$. That is, we indeed have $\epsilon<\infty$. Thus $e=\epsilon+\mu$ by Lemma 2.2. If $k \equiv \ell-\mu(\bmod e)$, say $k=\ell+j e-\mu=\ell+(j-1) e+\epsilon$, with $j \geq 1$, then for any zero $\beta$ of $f^{(\ell)}$,

$$
f^{(k)}(0)=f^{(\epsilon)}\left(f^{((j-1) e)}\left(f^{(\ell)}(\beta)\right)\right)=f^{(\epsilon)}\left(f^{((j-1) e)}(0)\right)=f^{(\epsilon)}(0) .
$$

Thus, any zero of $f^{(\ell)}$ is a pole of $f^{(k)}$. Suppose now that $k=\ell+j e+r-\mu$, with $j \geq 1$ and $1 \leq r<e$. If a zero $\beta$ of $f^{(\ell)}$ is a pole of $f^{(k)}$, then $f^{(k-\ell)}(0)=f^{(k)}(\beta)$, and so 0 is a pole of $f^{(k-\ell)}=f^{((j-1) e+\epsilon+r)}$. Since

$$
f^{((j-1) e+\epsilon)}(0)=f^{(\epsilon)}\left(f^{((j-1) e)}(0)\right)=f^{(\epsilon)}(0)
$$

0 is also a pole of $f^{((j-1) e+\epsilon)}$ and hence, by part (ii), $\operatorname{deg} g_{r}>\operatorname{deg} h_{r}$. This is a contradiction, since from (7) and the definition of $\mu, \operatorname{deg} g_{k} \leq \operatorname{deg} h_{k}$ for all $1 \leq k<$ $\mu+\min \{\epsilon, e\}=\mu+\epsilon=e$.

We may also determine facts about the degrees of iterates of $f$.

Lemma 2.4 Throughout, if $\min \{\mu, \nu\}<\infty$, define

$$
\delta=\left|\operatorname{deg} g_{\min \{\mu, \nu\}}-\operatorname{deg} h_{\min \{\mu, \nu\}}\right|
$$

and for a positive integer $j$, let $S_{j}$ and $T_{j}$ be respectively the degrees of the lowest order term in $g_{j}$ and $h_{j}$. Using the notation from Definition 2.1, we have

(i) If $v<\mu$, then for any integer $i \geq 1, \operatorname{deg} g_{i v}=d^{i v}$, and $\operatorname{deg} h_{i v}=d^{i v}-\delta^{i}$. Moreover, $\operatorname{deg} g_{j}=\operatorname{deg} h_{j}=d^{j}$ whenever $j \not \equiv 0(\bmod v)$.

(ii) If $\mu<v$ and $\epsilon=e=\infty$, then $\operatorname{deg} g_{j}=\operatorname{deg} h_{j}=d^{j}$ for all $j \neq \mu$.

(iii) Let $\mu<v, e<\infty$, and write $S_{e}=S$. Then $\operatorname{deg} g_{\mu+j}=d^{\mu+j}-\delta S_{j}$ and $\operatorname{deg} h_{\mu+j}=d^{\mu+j}-\delta T_{j}$ for any $j \geq 1$. If $j=i$ e for some integer $i \geq 1$, then $S_{j}=S^{i}$, and otherwise $S_{j}=0$. We moreover have the following.

(a) Suppose $e<\epsilon$. Then $T_{j}=0$ for all $j \geq 1$.

(b) Suppose $\epsilon<e$, and write $T_{\epsilon}=T$. Then $S=\delta T$. If $j=i e+\epsilon$ for some integer $i \geq 0$, then $T_{j}=\delta^{i} T^{i+1}$, and otherwise $T_{j}=0$.

Proof Throughout the proof, we will write a given iterate $f^{(k)}=u \circ F=P / Q$, and infer the degrees of its numerator and denominator via the equations (5) and (6).

For the first part, we proceed by induction on $i$. By definition, $\operatorname{deg} g_{j}=\operatorname{deg} h_{j}=$ $\operatorname{deg} f^{(j)}=d^{j}$ for $1 \leq j<v$, and we have $\operatorname{deg} g_{v}=d^{v}$ and $\operatorname{deg} h_{v}=d^{v}-\delta$. This proves the case $i=1$. Let $i \geq 1$ and suppose that $\operatorname{deg} g_{i v}=d^{i v}$ and $\operatorname{deg} h_{i v}=d^{i v}-\delta^{i}$. For an integer $1 \leq j \leq v$, set $u=f^{(j)}$ and $F=f^{(i v)}$. If $j<v$, we obtain 


$$
\begin{aligned}
\operatorname{deg} g_{i v+j} & =\operatorname{deg} h_{i v}\left(\operatorname{deg} f^{(j)}-\operatorname{deg} g_{j}\right)+\operatorname{deg} g_{i v} S_{j}+\operatorname{deg} f^{(i v)}\left(\operatorname{deg} g_{j}-S_{j}\right) \\
& =\left(d^{i v}-\delta\right)\left(d^{j}-d^{j}\right)+d^{i v} S_{j}+d^{i v}\left(d^{j}-S_{j}\right)=d^{i v+j},
\end{aligned}
$$

and similarly $\operatorname{deg} h_{i v+j}=d^{i v+j}$. When $j=v$, we get

$$
\begin{aligned}
\operatorname{deg} g_{(i+1) v} & =\operatorname{deg} h_{i v}\left(\operatorname{deg} f^{(v)}-\operatorname{deg} g_{v}\right)+\operatorname{deg} g_{i v} S_{v}+\operatorname{deg} f^{(i v)}\left(\operatorname{deg} g_{v}-S_{v}\right) \\
& =\left(d^{i v}-\delta\right)\left(d^{v}-d^{v}\right)+d^{i v} S_{v}+d^{i v}\left(d^{v}-S_{v}\right)=d^{(i+1) v},
\end{aligned}
$$

and

$$
\begin{aligned}
\operatorname{deg} h_{(i+1) v} & =\operatorname{deg} h_{i v}\left(\operatorname{deg} f^{(v)}-\operatorname{deg} h_{v}\right)+\operatorname{deg} g_{i v} T_{v}+\operatorname{deg} f^{(i v)}\left(\operatorname{deg} h_{v}-T_{v}\right) \\
& =\left(d^{i v}-\delta^{i}\right)\left(d^{v}-\left(d^{v}-\delta\right)\right)+d^{i v}\left(d^{v}-\delta\right)=d^{(i+1) v}-\delta^{i+1},
\end{aligned}
$$

as required. The second part follows from (7).

For the third part, setting $u=f^{(j)}$ and $F=f^{(\mu)}$ gives

$$
\begin{aligned}
\operatorname{deg} g_{j+\mu} & =\operatorname{deg} h_{\mu}\left(\operatorname{deg} f^{(j)}-\operatorname{deg} g_{j}\right)+\operatorname{deg} g_{\mu} S_{j}+\operatorname{deg} f^{(\mu)}\left(\operatorname{deg} g_{j}-S_{j}\right) \\
& =d^{\mu}\left(d^{j}-\operatorname{deg} g_{j}\right)+\left(d^{\mu}-\delta\right) S_{j}+d^{\mu}\left(\operatorname{deg} g_{j}-S_{j}\right)=d^{j+\mu}-\delta S_{j}
\end{aligned}
$$

and similarly $\operatorname{deg} h_{j+\mu}=d^{j+\mu}-\delta T_{j}$. If we put $u=f^{(e)}, F=f^{((i-1) e)}$, induction on $i$ with (4) shows that $S_{i e}=S_{e}^{i}=S^{i}$. Also, by Lemma $2.3(\mathrm{i})$, if $j \not \equiv 0(\bmod e)$, then no zero of $f^{(e)}$ (in particular 0) is a zero of $f^{(j)}$, and so $S_{j}=0$.

For the last part of the proof, we make use of Lemma 2.2. If $e<\epsilon$, we must have $\epsilon=\infty$, and so $T_{j}=0$ for all $j$, proving (a). On the other hand, if $\epsilon<e$, then $e=\epsilon+\mu$. Set $u=f^{(\mu)}$ and $F=f^{(\epsilon)}$ so that (4) gives $S=\delta T$, and thus $S_{i e}=\delta^{i} T^{i}$. We similarly obtain $T_{i e+\epsilon}=\delta^{i} T^{i+1}$. Finally, if $j$ is not equal to $i e+\epsilon$ for any integer $i \geq 0$, then $j \not \equiv \epsilon=e-\mu(\bmod e)$. Thus, by Lemma 2.3 (iv), no zero of $f^{(e)}$ (in particular 0 ) is a pole of $f^{(j)}$, and so $T_{j}=0$, proving (b).

Corollary 2.5 Suppose $\mu<v$ and $\epsilon<e<\infty$. Then for a positive integer $n$, deg $g_{n}<$ $\operatorname{deg} h_{n}$ if and only if $n \geq \mu$ and $n-\mu \equiv 0(\bmod e)$, and $\operatorname{deg} g_{n}>\operatorname{deg} h_{n}$ if and only if $n \geq \mu+\epsilon$ and $n-\mu \equiv \epsilon(\bmod e)$.

Proof By definition, we have $\operatorname{deg} g_{n}=\operatorname{deg} h_{n}$ for $n<\mu$, and $\operatorname{deg} g_{n}<\operatorname{deg} h_{n}$ for $n=\mu$. Suppose $n>\mu$, and write $n=\mu+j$, so $j=n-\mu$. Then from Lemma 2.4 (iii), $\operatorname{deg} g_{n}=d^{n}-\delta S_{j}$ and $\operatorname{deg} h_{n}=d^{n}-\delta T_{j}$. Hence $\operatorname{deg} g_{n}<\operatorname{deg} h_{n}$ if and only if $S_{j}>0$, which occurs precisely when $j=n-\mu \equiv 0(\bmod e)$ by Lemma 2.4 (iii).

On the other hand, $\operatorname{deg} g_{n}>\operatorname{deg} h_{n}$ if and only if $T_{j}>0$, and this happens precisely when $j=n-\mu \equiv \epsilon(\bmod e)$ by Lemma 2.4 (iii)(b).

We hence obtain the following result.

Lemma 2.6 Suppose $\mu<v$ and $\epsilon<\infty$ and let $1 \leq \ell<k$. Then

(i) A zero or pole of $f^{(\ell)}$ is a zero of $f^{(k)}$ if and only if it is a pole of $f^{(k-\mu)}$. In particular, if $k \leq \mu$, then no zero or pole of $f^{(\ell)}$ is a zero of $f^{(k)}$. 
(ii) A zero or pole of $f^{(\ell)}$ is a pole of $f^{(k)}$ if and only if it is a zero of $f^{(k-\epsilon)}$. In particular, if $k \leq \epsilon$, then no zero of pole of $f^{(\ell)}$ is a pole of $f^{(k)}$.

Proof Recall that since $\mu<v$ and $\epsilon<\infty$, we have $\epsilon<e<\infty$ and $e=\epsilon+\mu$ by Lemma 2.2.

For the first part, by Lemma 2.3 (i) we have that a zero of $f^{(\ell)}$ is a zero of $f^{(k)}$ if and only if $k \equiv \ell(\bmod e)$ (note that since $\ell<k$, this implies $k>\ell+e \geq 1+\epsilon+\mu>\mu)$. Then, by Lemma 2.3 (iv), a zero of $f^{(\ell)}$ is a pole of $f^{(k-\mu)}$ if and only if $k-\mu \equiv \ell-\mu$ $(\bmod e)$, which is an equivalent condition. From Lemma 2.3 (iii), a pole of $f^{(\ell)}$ is a zero of $f^{(k)}$ if and only if $\operatorname{deg} g_{k-\ell}<\operatorname{deg} h_{k-\ell}$. This occurs precisely when $k-\ell \geq \mu$ (and so $k \geq \mu)$ and $k-\ell-\mu \equiv 0(\bmod e)$ by Corollary 2.5. On the other hand, a pole of $f^{(\ell)}$ is a pole of $f^{(k-\mu)}$ if and only if $\operatorname{deg} g_{k-\ell-\mu}>\operatorname{deg} h_{k-\ell-\mu}$. By Corollary 2.5, this happens exactly when $k-\mu \equiv \ell(\bmod e)$, which is again equivalent.

For part (ii), by Lemma 2.3 (iv), a zero of $f^{(\ell)}$ is a pole of $f^{(k)}$ if and only if $k \equiv \ell-\mu(\bmod e)$. Since $e=\epsilon+\mu$, this is equivalent to $k-\epsilon \equiv \ell(\bmod e)$, which implies $k>\epsilon$, and is moreover the precise condition for a zero of $f^{(\ell)}$ to be a zero of $f^{(k-\epsilon)}$ by Lemma 2.3 (i). Furthermore, from Lemma 2.3 (ii), a pole of $f^{(\ell)}$ is a pole of $f^{(k)}$ if and only if $\operatorname{deg} g_{k-\ell}>\operatorname{deg} h_{k-\ell}$. By Corollary 2.5, this is equivalent to $k-\ell-\mu \equiv \epsilon(\bmod e)$. Again by Corollary 2.5 , this is equivalent to having $\operatorname{deg} g_{k-\ell-\epsilon}<\operatorname{deg} h_{k-\ell-\epsilon}$, which is in turn equivalent to the given pole of $f^{(\ell)}$ being a zero of $f^{(k-\epsilon)}$, by Lemma 2.3 (iii).

As we remarked in the introduction, in order to prove multiplicative independence for the iterates of $f$, it is clearly necessary to show that no iterate of $f$ is a monomial, that is, of the form $f(X)=a X^{ \pm d}$. We first look to a result of Silverman [21, Theorem 1]. Recall that two rational functions $\phi, \psi$ are linearly conjugate if there exists a rational function $u$ of degree 1 such that $\phi=u^{-1} \circ \psi \circ u$.

Lemma 2.7 Suppose there exists a positive integerk such that $f^{(k)} \in \mathbb{F}[X]$. Then either $f \in \mathbb{F}[X], f$ is separable and linearly conjugate to $1 / X^{d}$, or $f$ is not separable and $f(X)=L\left(X^{p^{\ell}}\right)$ for some $L \in \mathbb{F}(X)$ of degree 1 and integer $\ell \geq 0$.

Indeed, if no iterate of $f$ is a polynomial, then certainly none can be a monomial. In fact, in the case where $f$ is separable, we show that a rational function has a monomial iterate if and only if it is itself a monomial. This is not true however, when $f$ is not separable. For example, if $\mathbb{F}$ has characteristic 2 , then $f(X)=1+1 / X^{2}$ satisfies $f^{(2)}(X)=\frac{1}{X^{4}+1}$ and $f^{(3)}(X)=X^{8}$.

Note that in the case of characteristic 0 , some cases of the following can actually be viewed as a corollary of the stronger result [24, Theorem 1], which concerns the number of terms (monomials) of composite polynomials. The results of [24] are further extended to rational functions in [9].

Lemma 2.8 If $f \in \mathbb{F}(X)$ is neither a monomial, nor of the form $L\left(X^{p^{\ell}}\right)$ for some integer $\ell \geq 0$ and $L \in \mathbb{F}(X)$ of degree 1 , then $f^{(k)}$ is not a monomial for any $k \geq 1$.

Proof We begin with the case where $f \in \mathbb{F}[X]$ is a polynomial. First suppose $\mathbb{F}$ has zero characteristic. We proceed by induction on $k$. That is, suppose $\operatorname{deg} f \geq 2$, and 
that $f$ is not a monomial. Then the case where $k=1$ is trivial. If $f^{(k-1)}$ is not a monomial, we can write

$$
\begin{aligned}
f(X) & =a_{1} X^{d_{1}}+\cdots+a_{s} X^{d_{s}} \\
s & >1, d=d_{1}>\cdots>d_{s} \geq 0, a_{1}, \ldots, a_{s} \in \mathbb{F} \backslash\{0\},
\end{aligned}
$$

and

$$
\begin{aligned}
f^{(k-1)}(X) & =b_{1} X^{e_{1}}+\cdots+b_{t} X^{e_{t}} \\
t & >1, d^{k-1}=e_{1}>\cdots>e_{t} \geq 0, b_{1}, \ldots, b_{t} \in \mathbb{F} \backslash\{0\}
\end{aligned}
$$

Hence we have the following cases:

If $d_{s}=0, e_{t} \neq 0$, we have that

$$
\begin{aligned}
f^{(k)}(X) & =f\left(f^{(k-1)}(X)\right) \\
& =a_{1}\left(b_{1} X^{e_{1}}+\cdots+b_{t} X^{e_{t}}\right)^{d_{1}}+\cdots+a_{s}
\end{aligned}
$$

has constant term $a_{s} \neq 0$. Similarly, if $d_{s} \neq 0, e_{t}=0$,

$$
\begin{aligned}
f^{(k)}(X) & =f^{(k-1)}(f(X)) \\
& =b_{1}\left(a_{1} X^{d_{1}}+\cdots+a_{s} X^{d_{s}}\right)^{e_{1}}+\cdots+b_{t}
\end{aligned}
$$

has constant term $b_{t} \neq 0$. If $d_{s} \neq 0, e_{t} \neq 0$, then

$$
\begin{aligned}
f^{(k)}(X) & =f\left(f^{(k-1)}(X)\right) \\
& =a_{1}\left(b_{1} X^{e_{1}}+\cdots+b_{t} X^{e_{t}}\right)^{d_{1}}+\cdots+a_{s}\left(b_{1} X^{e_{1}}+\cdots+b_{t} X^{e_{t}}\right)^{d_{s}}
\end{aligned}
$$

has lowest order term $a_{s} b_{t}^{d_{s}} X^{d_{s} e_{t}} \neq 0$, since $a_{s} \neq 0, b_{t} \neq 0$. Finally, when $d_{s}=e_{t}=$ 0 , if $e_{2}>0$, we have

$$
\begin{aligned}
f^{(k)}(X) & =f\left(f^{(k-1)}(X)\right) \\
& =a_{1}\left(b_{1} X^{e_{1}}+b_{2} X^{e_{2}}+\cdots+b_{t}\right)^{d_{1}}+\cdots+a_{s} .
\end{aligned}
$$

In this case, the term in $X^{\left(d_{1}-1\right) e_{1}+e_{2}}$ has coefficient $d_{1} a_{1} b_{1}^{d_{1}-1} b_{2} \neq 0$, since we have $a_{1}, b_{1}, b_{2} \neq 0$, and $\mathbb{F}$ has 0 characteristic. Otherwise, $e_{2}=0$ and

$$
\begin{aligned}
f^{(k)}(X) & =f^{(k-1)}(f(X)) \\
& =b_{1}\left(a_{1} X^{d_{1}}+a_{2} X^{d_{2}}+\cdots+a_{s}\right)^{e_{1}}+b_{2} .
\end{aligned}
$$

Similarly, the term in $X^{\left(e_{1}-1\right) d_{1}+d_{2}}$ has coefficient $e_{1} b_{1} a_{1}^{e_{1}-1} a_{2} \neq 0$. That is, in all cases $f^{(k)}$ is not a monomial, and we are done. 
Now, suppose $\mathbb{F}$ has positive characteristic $p$, and that $f^{(k)}$ is monomial, say of the form $c X^{d^{k}}$ with $c \in \mathbb{F} \backslash\{0\}$, for some $k>1$. We can write

$$
f(X)=a_{1} X^{d_{1} p^{\ell}}+\cdots+a_{t} X^{d_{t} p^{\ell}}+b
$$

where $a_{1}, \ldots, a_{t} \in \mathbb{F} \backslash\{0\}, b \in \mathbb{F}, t \geq 1, \ell \geq 0, d_{1}>\cdots>d_{t} \geq 1$, and $p \nmid \operatorname{gcd}\left(d_{1}, \ldots, d_{t}\right)$.

Here, the degree of $f$ is $d=d_{1} p^{\ell}$. Denote $r=p^{\ell}$ and let

$$
\begin{aligned}
v(X) & =a_{1} X^{d_{1}}+\cdots+a_{t} X^{d_{t}}+b \\
w_{i}(X) & =a_{1}^{r^{-i}} X^{d_{1}}+\cdots+a_{t}^{r^{-i}} X^{d_{t}}+b^{r^{-i}}, i \geq 1 .
\end{aligned}
$$

Since $r^{i}$ is a power of $p$, we have for any $i \geq 1$

$$
\left(w_{i}(X)\right)^{r^{i}}=a_{1} X^{d_{1} r^{i}}+\cdots+a_{t} X^{d_{t} r^{i}}+b=v\left(X^{r^{i}}\right) .
$$

Hence

$$
\begin{aligned}
f(X) & =v\left(X^{r}\right), \\
f^{(2)}(X) & \left.=v\left(v\left(X^{r}\right)^{r}\right)\right)=v\left(\left(w_{1}(X)\right)^{r^{2}}\right)=\left(w_{2} \circ w_{1}(X)\right)^{r^{2}} . \\
& \vdots \\
f^{(k)}(X) & =\left(w_{k} \circ w_{k-1} \circ \cdots \circ w_{1}(X)\right)^{r^{k}}, \quad k \geq 1 .
\end{aligned}
$$

Hence we have

$$
w_{k} \circ w_{k-1} \circ \cdots \circ w_{1}(X)=c_{0} X^{d_{1}^{k}}
$$

where $c_{0}=c^{r^{-k}} \neq 0$, since $c \neq 0$. Differentiating then gives

$$
\begin{aligned}
& w_{k}^{\prime}\left(w_{k-1} \circ \cdots \circ w_{1}(X)\right) \cdot w_{k-1}^{\prime}\left(w_{k-2} \circ \cdots \circ w_{1}(X)\right) \cdots w_{2}^{\prime}\left(w_{1}(X)\right) \cdot w_{1}^{\prime}(X) \\
& =d_{1}^{k} c_{0} X^{d_{1}^{k}-1} .
\end{aligned}
$$

Since $p \nmid \operatorname{gcd}\left(d_{1}, \ldots, d_{t}\right), w_{i}^{\prime} \neq 0$ for all $i \geq 1$. Thus, the polynomial on the left hand side of (8) is not zero. So $p \nmid d_{1}$, as otherwise the right hand side would be zero. Since $d_{1}^{k} c_{0} \neq 0$, the Eq. (8) implies that $w_{1}^{\prime}(X)$ divides $X^{d_{1}^{k}-1}$. Therefore $w_{1}^{\prime}$ is a monomial. Since $p \nmid d_{1}$, we must have $p \mid d_{i}$ for $2 \leq i \leq t$. Hence

$$
w_{i}^{\prime}(X)=d_{1} a_{1}^{-r^{i}} X^{d_{1}-1}, \quad i \geq 1
$$

and so $w_{2}^{\prime}\left(w_{1}(X)\right)=d_{1} a_{1}^{-r^{2}}\left(w_{1}(X)\right)^{d_{1}-1}$ is also a factor of $X^{d_{1}^{k}-1}$. If $d_{1}>1$, then $w_{1}$ is a monomial and hence $f$ must also be a monomial. If $d_{1}=1$, then $d_{1}>\cdots>d_{t} \geq 1$ implies that $t=1$. Therefore $f$ is a binomial of the form $a X^{p^{\ell}}+b$. 
Now, suppose $f \notin \mathbb{F}[X]$, and that $f^{(k)}$ is a monomial for some $k \geq 1$. Then in particular, some iterate of $f$ is a polynomial.

If $f$ is separable, then by Lemma $2.7, f$ is linearly conjugate to $1 / X^{d}$. That is, $f$ has the form

$$
f(X)=a+\frac{b}{(X-a)^{d}}, \quad a, b \in \mathbb{F} .
$$

Then $f^{(2)}(X)=a+b^{1-d}(X-a)^{d^{2}} \in \mathbb{F}[X]$, which is a monomial if and only if $a=0$, in which case $f$ is a monomial. Suppose $a \neq 0$. Since $f$ is separable, $d \neq p^{\ell}$ for any $\ell>0$, and so, since we have already proved the result for polynomials, no iterate of $f^{(2)}$ is a monomial. That is, $f^{(k)}$ is not a monomial for any even $k \geq 2$ unless $f$ is a monomial. Moreover, we have in this case $v=2<\mu$, so by Lemma 2.4 (i), $\operatorname{deg} g_{k}=\operatorname{deg} h_{k}$, and so $f^{(k)}$ is not a monomial, for all odd $k$.

Finally, if $f$ is not separable, then by Lemma $2.7, f^{(k)}$ is not a polynomial, and hence is not a monomial, for any $k \geq 1$ unless $f$ is of the form $L\left(X^{p^{\ell}}\right)$ for some $L \in \mathbb{F}(X)$ of degree 1 .

We can now prove Theorem 1.1. Recall that we write $f^{(k)}=g_{k} / h_{k}$ in lowest terms, and define $\delta, S_{k}$, and $T_{k}$ as in Lemma 2.4, again setting $S=S_{e}$ and $T=T_{\epsilon}$ where applicable. Now, where $\Psi(n)$ is defined as in (1), noting that $\mathbb{F}(X)$ is a unique factorisation domain, any zeros or poles of $f^{(n)}$ which can not be found in previous iterates will contribute to the value of $\Psi(n)$ counting multiplicity, since $k_{n} \neq 0$.

We first consider the case where $v<\mu$. Then $\operatorname{deg} g_{k} \geq \operatorname{deg} h_{k}$ for all $k$ by Lemma 2.4 (i). Hence $\operatorname{gcd}\left(g_{n}, h_{k}\right)=1$ for any $k<n$ by Lemma 2.3 (iii). Moreover, if $n \leq e$, then $\operatorname{gcd}\left(g_{n}, g_{k}\right)=1$ for any $k<n$, by Lemma 2.3 (i). In this case, we have $\Psi(n) \geq \operatorname{deg} g_{n}=d^{n}$. Suppose $e<\infty$ and $n>e$. Then for $k<n$, a zero of $f^{(k)}$ is a zero of $f^{(n)}$ if and only if $k \equiv n(\bmod e)$ by Lemma 2.3. In this case we also have $k \equiv n-e(\bmod e)$, and so such a zero must also be a zero of $f^{(n-e)}$. Write $u=f^{(e)}$ and $F=f^{(n-e)}$, so (4) gives $g_{n}=g_{n-e}^{S} q$, where $S>0$ and $\operatorname{gcd}\left(q, g_{n-e}\right)=1$. Since $f^{(e)}$ is not a monomial by Lemma 2.8 , we have $S<d^{e}$, and so $\Psi(n) \geq \operatorname{deg} q=d^{n}-S d^{n-e} \geq d^{n-e}$.

Now, suppose $\mu<v$. If $e=\epsilon=\infty$ or $e<\epsilon$, then by Lemma 2.4 (ii) and (iii)(a), $\operatorname{deg} g_{j} \leq \operatorname{deg} h_{j}=d^{j}$ for all $j \geq 1$, and so $\operatorname{gcd}\left(h_{k}, h_{n}\right)=1$ for all $1 \leq k<n$ by Lemma 2.3 (ii). Moreover, $\operatorname{gcd}\left(g_{k}, h_{n}\right)=1$ for all $1 \leq k<n$ by Lemma 2.3 (iv). Hence $\Psi(n) \geq \operatorname{deg} h_{n}=d^{n}$. Suppose $\epsilon<\infty$. Then $\mu<\epsilon<e<\infty$ by Lemma 2.2. Moreover, if $n \leq \epsilon, \operatorname{gcd}\left(g_{k}, h_{n}\right)=\operatorname{gcd}\left(h_{k}, h_{n}\right)=1$ by Lemma 2.6 (ii), and thus we again have $\Psi(n) \geq \operatorname{deg} h_{n}=d^{n}$. We hence assume that $\mu \leq \epsilon<n<\infty$.

We now split into a further two cases. Firstly, suppose that $\operatorname{deg} g_{\mu}>0$, so that $\delta<d^{\mu}$. Since $e=\mu+\epsilon>\mu$, we do not have $\mu \equiv 0(\bmod e)$, and so $S_{\mu}=0$ by Lemma 2.4 (iii). Hence, where $u=f^{(\mu)}$ and $F=f^{(n-\mu)}$, (4) gives

$$
g_{n}=h_{n-\mu}^{\delta} q,
$$

for some polynomial $q$ relatively prime to $h_{n-\mu}$. From Lemma 2.6 (i), any zero or pole of a previous iterate $f^{(k)}, 1 \leq k<n$, which is also a zero of $f^{(n)}$, must be a root of 
$h_{n-\mu}$. Hence $\Psi(n) \geq \operatorname{deg} q$, and so we aim to bound $\operatorname{deg} q$ from below. If $n=\mu+i e$ for some integer $i \geq 1$, then $n-\mu=\mu+(i-1) e+\epsilon$, and so by Lemma 2.4 (iii)(b),

$$
\begin{aligned}
\delta \operatorname{deg} h_{n-\mu}+\left(\operatorname{deg} g_{\mu}\right) d^{n-\mu} & =\delta\left(d^{n-\mu}-\delta^{i} T^{i}\right)+\left(d^{\mu}-\delta\right) d^{n-\mu} \\
& =d^{n}-\delta^{i+1} T^{i}=\operatorname{deg} g_{n} .
\end{aligned}
$$

Otherwise, $n-\mu \not \equiv 0(\bmod e)$, and so $\operatorname{deg} g_{n} \geq \operatorname{deg} h_{n}$ by Corollary 2.5. That is, $\operatorname{deg} g_{n}=d^{n}$, and so

$$
\delta \operatorname{deg} h_{n-\mu}+\left(\operatorname{deg} g_{\mu}\right) d^{n-\mu} \leq \delta d^{n-\mu}+\left(d^{\mu}-\delta\right) d^{n-\mu}=d^{n}=\operatorname{deg} g_{n} .
$$

Hence from (9)

$$
\operatorname{deg} q=\operatorname{deg} g_{n}-\delta \operatorname{deg} h_{n-\mu} \geq\left(\operatorname{deg} g_{\mu}\right) d^{n-\mu} \geq d^{n-\mu},
$$

and therefore $\Psi(n) \geq \operatorname{deg} q \geq d^{n-\mu}$.

On the other hand, where $\operatorname{deg} g_{\mu}=0$ and correspondingly $\delta=d^{\mu}$, we set $u=$ $f^{(\epsilon)}$, and $F=f^{(n-\epsilon)}$. If $\epsilon=\mu$, then by definition $\operatorname{deg} g_{\epsilon}<\operatorname{deg} h_{\epsilon}$. Otherwise $0<\epsilon-\mu<\epsilon$, so $\epsilon-\mu \not \equiv 0, \epsilon(\bmod e)$, and thus $\operatorname{deg} g_{\epsilon}=\operatorname{deg} h_{\epsilon}$ by Corollary 2.5. Hence, in (4), $f^{(n)}=h_{n-\epsilon}^{m-l} g_{n-\epsilon}^{-T} q / r$, where $m=\operatorname{deg} h_{e} \geq \operatorname{deg} g_{e}=l$. That is,

$$
h_{n}=g_{n-\epsilon}^{T} r,
$$

where $r$ is a polynomial relatively prime to $g_{n-\epsilon}$. From Lemma 2.6 (ii), any zero or pole of a previous iterate $f^{(k)}, 1 \leq k<n$, which is also a pole of $f^{(n)}$, must be a root of $g_{n-\epsilon}$. Hence $\Psi(n) \geq \operatorname{deg} r=\operatorname{deg} h_{n}-T \operatorname{deg} g_{n-\epsilon}$. Note that $T<d^{\epsilon}$, as if this were not the case, by Lemma 2.4 (iii) we would have

$$
\operatorname{deg} h_{\mu+\epsilon}=d^{\mu+\epsilon}-\delta T=d^{\mu+\epsilon}-d^{\mu} d^{\epsilon}=0,
$$

and $S_{\mu+\epsilon}=S_{e}=\delta T=d^{\mu} d^{\epsilon}$, which implies that $f^{(\mu+\epsilon)}$ is a monomial, contradicting Lemma 2.8. In particular, this means that

$$
d^{n}-T d^{n-\epsilon} \geq d^{n-\epsilon}
$$

Hence, if $n=\mu+i e+\epsilon$ for some integer $i \geq 0$, then $n-\epsilon=\mu+i e$, so by Lemma 2.4 (iii), (10) and (11), we have

$$
\operatorname{deg} r=d^{n}-\delta^{i+1} T^{i+1}-T\left(d^{n-\epsilon}-\delta^{i+1} T^{i}\right)=d^{n}-T d^{n-\epsilon} \geq d^{n-\epsilon} .
$$

Otherwise, $n-\mu \not \equiv \epsilon(\bmod e)$, and so $\operatorname{deg} g_{n} \leq \operatorname{deg} h_{n}$ by Corollary 2.5. That is, $\operatorname{deg} h_{n}=d^{n}$, and so from (10) and (11)

$$
\operatorname{deg} r=d^{n}-T \operatorname{deg} g_{n-\epsilon} \geq d^{n}-T d^{n-\epsilon} \geq d^{n-\epsilon} .
$$

We conclude that $\Psi(n) \geq \operatorname{deg} r \geq d^{n-\epsilon}$, completing the proof. 


\section{Proof of Theorem 1.3}

Recall the polynomial $A B C$-theorem (proved first by Stothers [23], then independently by Mason [14] and Silverman [22]).

Lemma 3.1 Let $\mathbb{F}$ be a field and let $A, B, C \in \mathbb{F}[X]$ be relatively prime polynomials such that $A+B+C=0$ and not all of $A, B$ and $C$ have vanishing derivative. Then

$$
\max \{\operatorname{deg} A, \operatorname{deg} B, \operatorname{deg} C\} \leq \operatorname{deg} \operatorname{rad}(A B C)-1,
$$

where, for $f \in \mathbb{F}[X] \operatorname{rad}(f)$ is the product of the distinct monic irreducible factors of $f$.

We use this to obtain a version of part of the main result of [8]. Namely, we give a lower bound for the number of distinct zeros of a composite polynomial.

Lemma 3.2 Let $f=g \circ h \in \mathbb{F}[X]$, where $g, h \in \mathbb{F}[X]$, h has non-vanishing derivative, and $\mathrm{z}(g) \geq 2$. Then

$$
\mathrm{z}(f) \geq \gamma(g) \operatorname{deg} h+1
$$

where $\gamma$ is defined as in Theorem 1.3.

Proof If $\operatorname{deg} h=1$, then clearly $\mathrm{z}(f)=\mathrm{z}(g)$. Since $\mathrm{z}(g) \geq 2$, we have

$$
\gamma(g) \operatorname{deg} h+1=\max \{\mathrm{z}(g)-1,2\} \leq \mathrm{z}(g)=\mathrm{z}(f)
$$

so assume $\operatorname{deg} h \geq 2$.

In the characteristic 0 case, the result is [8, Main Theorem (i)]. When the characteristic is positive, we proceed in much the same vein. Write

$$
f(X)=\prod_{i=1}^{n}\left(X-\alpha_{i}\right)^{f_{i}}, \quad g(X)=\prod_{j=1}^{t}\left(X-\beta_{j}\right)^{k_{j}},
$$

where the $\alpha_{i}$ and $\beta_{j}$ are respectively the distinct roots of $f$ and $g$ in an algebraic closure of $f$. Then

$$
f(X)=g(h(X))=\prod_{j=1}^{t}\left(h(X)-\beta_{j}\right)^{k_{j}} .
$$

For $\beta_{i} \neq \beta_{j}$, the factors $h(X)-\beta_{i}$ and $h(X)-\beta_{j}$ have no zeros in common, so $t \leq n$, and there exists a partition of $\{1, \ldots, n\}$ into disjoint subsets $S_{\beta_{1}}, \ldots, S_{\beta_{t}}$, such that

$$
h(X)-\beta_{j}=p_{j}(X):=\prod_{m \in S_{\beta_{j}}}\left(X-\alpha_{m}\right)^{l_{m}},
$$


with $l_{m} k_{m}=f_{m}$, for every $j=1, \ldots, t$. Since $t=\mathrm{z}(g)>1$, we can take $1 \leq i<$ $j \leq t$, and obtain $h(X)=\beta_{i}+p_{i}(X)=\beta_{j}+p_{j}(X)$. That is,

$$
\left(\beta_{i}-\beta_{j}\right)+p_{i}+\left(-p_{j}\right)=0
$$

where the polynomials on the left-hand side are relatively prime, and in particular, since $h$ has non-vanishing derivative, so does $p_{i}$. Thus, applying Lemma 3.1, we have

$$
\begin{gathered}
\max \left\{\operatorname{deg}\left(\beta_{i}-\beta_{j}\right), \operatorname{deg} p_{i}, \operatorname{deg}\left(-p_{j}\right)\right\}=\operatorname{deg} h \\
\leq \operatorname{deg} \operatorname{rad}\left(\left(\beta_{j}-\beta_{i}\right) p_{i} p_{j}\right)-1 \leq n-1
\end{gathered}
$$

Therefore $n=\mathrm{z}(f) \geq \operatorname{deg} h+1$.

We now prove Theorem 1.3. Suppose $f \in \mathbb{F}[X]$ has non-vanishing derivative. Then for any positive integer $n$,

$$
\frac{d}{d X} f^{(n)}(X)=f^{\prime}\left(f^{(n-1)}(X)\right) \cdot f^{\prime}\left(f^{(n-2)}(X)\right) \cdots f^{\prime}(f(X)) \cdot f^{\prime}(X) \neq 0 .
$$

We can hence apply Lemma 3.2 to obtain $\mathrm{z}\left(f^{(n)}\right) \geq \gamma(f) d^{n-1}+1$. As in the proof of Theorem 1.1, any zeros of $f^{(n)}$ which cannot be found in previous iterates will contribute to the value of $Z(n)$, but this time without multiplicity. If $n \leq e$, then $\operatorname{gcd}\left(f^{(k)}, f^{(n)}\right)=1$ for all $1 \leq k<n$ by Lemma 2.3 (i), and so $Z(n) \geq \mathrm{z}\left(f^{(n)}\right) \geq$ $\gamma(f) d^{n-1}+1$. Suppose that $e<n<\infty$, and write

$$
f^{(e)}(X)=X^{S} \phi(X), \quad S \geq 1, \phi(0) \neq 0 .
$$

Note that any zeros of $f^{(n)}$ which are common with a previous iterate belong to $f^{(n-e)}$ by Lemma 2.3 (i). Now,

$$
f^{(n)}(X)=f^{(e)}\left(f^{(n-e)}(X)\right)=\left(f^{(n-e)}(X)\right)^{S} \phi\left(f^{(n-e)}(X)\right) .
$$

If $e>1$, then $\mathrm{z}\left(f^{(e)}\right) \geq d^{e-1}+1>2$, and otherwise $\mathrm{z}\left(f^{(e)}\right)>2$ by assumption. Hence $\mathrm{z}(\phi)>1$, and so by Lemma 3.2, $Z(n) \geq \mathrm{z}\left(\phi\left(f^{(n-e)}\right)\right) \geq \gamma(\phi) d^{n-e}+1 \geq$ $d^{n-e}+1$.

\section{Proof of Theorem 1.4}

If $f \in \mathbb{F}[X]$, this is the main result of [19], so assume otherwise, in which case we define $s=\lfloor(n-1) / 2\rfloor$. Recall the following lower bound from Lambe [12], on the number of solutions to a linear Diophantine inequality:

Lemma 4.1 Suppose that $m$ and $x_{0}, \ldots, x_{r-1}$ are positive integers such that $\operatorname{gcd}\left(x_{0}, \ldots, x_{r-1}\right)=1$. Then the number of non-negative integer solutions $a_{0}, \ldots, a_{r-1}$ to the inequality 


$$
\sum_{i=0}^{r-1} a_{i} x_{i} \leq m
$$

is at least

$$
\left(\begin{array}{c}
m+r \\
r
\end{array}\right) \prod_{i=0}^{r-1} \frac{1}{x_{i}}
$$

with equality when $x_{0}=\cdots=x_{r-1}=1$.

Now, set $m=\bar{n}$. Since $\alpha$ is a root of $X^{m} h(X)-g(X)$, we have $\alpha^{m}=f(\alpha)$. As $m$ is a power of $q$, applying the Frobenius automorphism iteratively gives

$$
\alpha^{m^{i}}=f^{(i)}(\alpha), i \geq 0
$$

Consider the set

$$
S=\left\{\sum_{i=0}^{t-1} a_{i} m^{i}: \sum_{i=0}^{t-1} a_{i} d^{i} \leq s, \quad a_{i} \geq 0\right\}
$$

Suppose $a \in S$ has two representations $a=\sum_{i=0}^{t-1} a_{i} m^{i}=\sum_{i=0}^{t-1} b_{i} m^{i}$. For each $i$,

$$
0 \leq a_{i}, b_{i} \leq s<n \leq m,
$$

so $\sum_{i=0}^{t-1} a_{i} m^{i}$ and $\sum_{i=0}^{t-1} b_{i} m^{i}$ are both base- $m$ expansions for $a$. Hence $a_{i}=b_{i}$ for each $i$, and so $S$ has order equal to the number of non-negative integer solutions to the inequality

$$
\sum_{i=0}^{t-1} a_{i} m^{i} \leq s
$$

Thus, by Lemma 4.1,

$$
\# S \geq\left(\begin{array}{c}
s+t \\
t
\end{array}\right) \prod_{i=0}^{t-1} \frac{1}{d^{i}}
$$

We will show that the powers $\alpha^{a}$, with $a \in S$, are distinct in $\mathbb{F}_{q^{n}}$, so from Lemma 4.1, $\alpha$ has order at least \#S.

Suppose that there exist integers $a, b$ in $S$ such that $\alpha^{a}=\alpha^{b}$. Writing $a=$ $\sum_{i=0}^{t-1} a_{i} m^{i}$ and $b=\sum_{i=0}^{t-1} b_{i} m^{i}$, we have

$$
\prod_{i=0}^{t-1}\left(\alpha^{m^{i}}\right)^{a_{i}}=\prod_{i=0}^{t-1}\left(\alpha^{m^{i}}\right)^{b_{i}}
$$


The equation (12) then gives

$$
\prod_{i=0}^{t-1}\left(f^{(i)}(\alpha)\right)^{a_{i}}=\prod_{i=0}^{t-1}\left(f^{(i)}(\alpha)\right)^{b_{i}} .
$$

Let

$$
k_{1}(X)=\prod_{a_{i}>b_{i}} g_{i}(X)^{a_{i}-b_{i}} \prod_{a_{i}<b_{i}} h_{i}(X)^{b_{i}-a_{i}}
$$

and

$$
k_{2}(X)=\prod_{a_{i}<b_{i}} g_{i}(X)^{b_{i}-a_{i}} \prod_{a_{i}>b_{i}} h_{i}(X)^{a_{i}-b_{i}} .
$$

Then $k_{1}(\alpha)=k_{2}(\alpha)$. Since $\alpha$ has degree $n$ and $k_{1}$ and $k_{2}$ have degree at most

$$
\sum_{i=0}^{t-1} \max \left\{a_{i}, b_{i}\right\} d^{i} \leq 2 s \leq n-1,
$$

we have $k_{1}(X)=k_{2}(X)$. Thus $\prod_{i=0}^{t-1}\left(f^{(i)}(X)\right)^{a_{i}-b_{i}}=1$. Then $a_{i}-b_{i}=0$ for each $i$ by Corollary 1.2, and hence $a=b$.

In light of Theorem 1.4, we wish to determine whether such a pair $(g, h)$ of suitable polynomials always exists for all $n$. If this is so, we can construct a reliable algorithm for finding elements of high order in $\mathbb{F}_{q^{n}}$. Namely, checking $X^{\bar{n}} h(X)-g(X)$ for irreducible factors of degree $n$, for each appropriate pair $(g, h) \in \mathbb{F}_{q}[X]^{2}$. The case where $h(X)=1$ is considered in [10], where it is reasonably conjectured, but not proved, that for every $n$, there exists $g \in \mathbb{F}_{q}[X]$ with $\operatorname{deg} g \leq 2 \log _{q} n$, such that $X^{\bar{n}}-g(X)$ has an irreducible factor of degree $n$.

For our more general situation, we make the following weaker conjecture,

Conjecture 4.2 Suppose $n \geq 1$, and let $T$ be the set of pairs $(g, h) \in \mathbb{F}_{q}[X]^{2}$ of degree not exceeding $d:=\left\lceil 2 \log _{q} n\right\rceil$ such that $f=g / h$ satisfies the conditions from Corollary 1.2. Then there exists $(g, h) \in T$ such that $X^{\bar{n}} h(X)-g(X)$ has an irreducible factor of degree $n$.

To give some evidence for this conjecture, we first obtain a rough lower bound for the order of $T$. See [2] for the next lemma, regarding the probability that two polynomials in $\mathbb{F}_{q}[X]$ are relatively prime.

Lemma 4.3 Let $g$ and $h$ be randomly chosen from the set of polynomials in $\mathbb{F}_{q}[X]$ of degree $a$ and $b$ respectively, where $a$ and $b$ are not both zero. Then the probability that $g$ and $h$ are relatively prime is $1-1 / q$. 
Clearly, every pair $(g, h) \in \mathbb{F}_{q}[X]^{2}$ with $\operatorname{deg} g=d$, deg $h=d-1$ and $\operatorname{gcd}(g, h)=$ 1 is an element of $T$. Thus, Lemma 4.3. gives

$$
\begin{aligned}
\# T & \geq\left(1-\frac{1}{q}\right) \cdot(q-1) q^{d} \cdot(q-1) q^{d-1} \\
& \geq \frac{(q-1)^{3}}{q^{2}} q^{4 \log _{q} n}=\frac{(q-1)^{3}}{q^{2}} n^{4} .
\end{aligned}
$$

Now, consider the following result from [10]:

Lemma 4.4 Let $P_{q}(m, n)$ be the probability of a random polynomial in $\mathbb{F}_{q}[X]$ of degree $m \geq n$ having at least one irreducible factor of degree $n$. Then

$$
P_{q}(m, n) \sim \frac{1}{n}, \quad \text { as } n \rightarrow \infty,
$$

uniformly for $q$ and $m \geq n$.

If we model $X^{\bar{n}} h(X)-g(X)$ as a random polynomial in $\mathbb{F}_{q}[X]$ for each $(g, h) \in T$, Lemma 4.4, in conjunction with (13), suggests that for large $n$, we expect on the order of $n^{3}$ pairs $(g, h) \in T$ such that $X^{\bar{n}} h(X)-g(X)$ has an irreducible factor of degree $n$. Thus it is plausible that at least one such pair exists.

\section{Proof of Theorem 1.5}

We now restrict the field $\mathbb{F}$ to having characteristic 0 . The key tool of this section is Lemma 3.1, and so the results could perhaps be extended to characteristic $p$, given stronger conditions to ensure that one of the polynomials $A, B$ or $C$, to which we apply the theorem, has non-vanishing derivative.

We now prove Theorem 1.5. Suppose $F_{1}(X, u(X)), \ldots, F_{n}(X, u(X))$ are multiplicatively dependent, and and assume that no proper subset of these is also multiplicatively dependent, as we can remove functions until this is the case. Then every zero and pole of $F_{i}$ for $1 \leq i \leq n$ must be a zero or pole of $F_{j}$ for some $j \neq i$. This is because otherwise we would require $k_{i}=0$ in the equation

$$
\prod_{\ell=1}^{n} F_{\ell}(X, u(X))^{k_{\ell}}=1
$$

and hence the proper subset $\left\{F_{\ell}(X, u(X)): 1 \leq \ell \leq n, \ell \neq i\right\}$ would be multiplicatively dependent. Hence, if $\alpha$ is a zero or pole or $F_{i}(X, u(X))$, there exists $j \neq i$ such that $F_{i}(\alpha, Y)$ and $F_{j}(\alpha, Y)$ have the common zero or pole $u(\alpha)$, giving $R_{i j}(\alpha)=0$. Thus, any zero or pole of $F_{i}(X, u(X))$ for $1 \leq i \leq n$ is a zero of $\prod_{1 \leq i<j} \prod_{i<j \leq n} R_{i j}$. In particular, since for all $i \neq j, R_{i j}$ is not identically zero, we have

$$
\operatorname{deg} \operatorname{rad} \prod_{i=1}^{n} G_{i}(X, u(X)) H_{i}(X, u(X)) \leq \sum_{1 \leq i<j} \sum_{i<j \leq n} \operatorname{deg} R_{i j}=E .
$$


Now, for $1 \leq i \leq n$, write

$$
F_{i}(X, Y)=\frac{G_{i}(X, Y)}{H_{i}(X, Y)}=\frac{\sum_{\nu=0}^{e_{i}} g_{i, v}(X) Y^{\nu}}{\sum_{\nu=0}^{e_{i}} h_{i, \nu}(X) Y^{\nu}}
$$

and assume, without loss of generality, that $g_{i, e_{i}}$ is not identically zero (if it is, we can replace $G_{i}$ with $H_{i}$, and $g_{i, e_{i}}$ with $h_{i, e_{i}}$ in the following definitions). For $1 \leq i<j \leq n$, define

$$
P(X)=g_{i, e_{i}}(X) G_{j}(X, u(X)), \quad Q(X)=g_{j, e_{j}}(X) u(X)^{e_{j}-e_{i}} G_{i}(X, u(X)),
$$

and $D_{i j}(X)=\operatorname{gcd}(P(X), Q(X))$. Then set

$$
A(X)=\frac{P(X)}{D_{i j}(X)}, \quad B(X)=-\frac{Q(X)}{D_{i j}(X)}, \quad C(X)=-(A(X)+B(X)) .
$$

Then $A, B$, and $C$ are relatively prime polynomials with $A+B+C=0$.

Suppose $\operatorname{deg} u>2 d_{n}$. By construction, $P$ and $Q$ have the same degree and same leading coefficient, and hence we have $P \mid Q$ if and only if $P=Q$. If $P=Q$, then

$$
\begin{aligned}
P(X)-Q(X)= & \sum_{\nu=e_{j}-e_{i}}^{e_{j}}\left(g_{i, e_{i}}(X) g_{j, v}(X)-g_{j, e_{j}}(X) g_{i, v-e_{j}+e_{i}}(X)\right) u(X)^{v} \\
& +\sum_{\nu=0}^{e_{j}-e_{i}-1} g_{i, e_{i}}(X) g_{j, v}(X) u(X)^{v}=0 .
\end{aligned}
$$

Since deg $u>2 d_{n}$, the term in $u(X)^{v}$ in the above expression contains monomials in $X$ of degree between $v \operatorname{deg} u$ and $v \operatorname{deg} u+2 d_{n}<(v+1) \operatorname{deg} u$. Thus there can be no cancellation between these terms, and so

$$
g_{i, e_{i}}(X) g_{j, v}(X)-g_{j, e_{j}}(X) g_{i, v-e_{j}+e_{i}}(X)=0, \quad e_{j}-e_{i} \leq v \leq e_{j},
$$

and

$$
g_{i, e_{i}}(X) g_{j, v}(X)=0, \quad 0 \leq v<e_{j}-e_{i} .
$$

We conclude that in fact

$$
g_{i, e_{i}}(X) G_{j}(X, Y)=g_{j, e_{j}}(X) Y^{e_{j}-e_{i}} G_{i}(X, Y)
$$

but $R_{i j} \not \equiv 0$ implies that $\operatorname{gcd}\left(G_{j}(X, Y), G_{i}(X, Y)\right)=1$, and so we must have

$$
G_{j}(X, Y) \mid g_{j, e_{j}}(X) Y^{e_{j}-e_{i}} .
$$


This is impossible as $G_{j}(X, Y)$ has degree $e_{j}>e_{j}-e_{i}$ in $Y$. Therefore $P \nmid Q$, and so $\operatorname{deg} D_{i j}<\operatorname{deg} P$ gives

$$
\operatorname{deg} A=\operatorname{deg} P-\operatorname{deg} D_{i j}=\operatorname{deg} g_{i, e_{i}}+\operatorname{deg} g_{j, e_{j}}+e_{j} \operatorname{deg} u-\operatorname{deg} D_{i j}>0 .
$$

Thus $A$ has non-vanishing derivative. Moreover, in $C$, the term in $u(X)^{e_{j}}$ cancels out, giving

$$
\begin{aligned}
\operatorname{deg} C \leq & \left(e_{j}-1\right) \operatorname{deg} u \\
& +\max \left\{\operatorname{deg} g_{i, e_{i}}+\operatorname{deg} g_{j, e_{j}-1}, \operatorname{deg} g_{j, e_{j}}+\operatorname{deg} g_{i, e_{i}-1}\right\}-\operatorname{deg} D_{i j}
\end{aligned}
$$

Therefore, we have by Lemma 3.1 and (16),

$$
\begin{aligned}
\operatorname{deg} A & =\operatorname{deg} g_{i, e_{i}}+\operatorname{deg} g_{j, e_{j}}+e_{j} \operatorname{deg} u-\operatorname{deg} D_{i j} \\
& \leq \max \{\operatorname{deg} A, \operatorname{deg} B, \operatorname{deg} C\} \\
& \leq \operatorname{deg} \operatorname{rad} A B C-1 \\
& \leq \operatorname{deg} \operatorname{rad} G_{i}(X, u(X)) G_{j}(X, u(X))+\operatorname{deg} g_{i, e_{i}}+\operatorname{deg} g_{j, e_{j}}+\operatorname{deg} C-1 .
\end{aligned}
$$

From (15), $\operatorname{deg} \operatorname{rad} G_{i}(X, u(X)) G_{j}(X, u(X)) \leq E$, and so (17) gives

$$
\begin{aligned}
e_{j} \operatorname{deg} u-\operatorname{deg} D_{i j} \leq & E+\left(e_{j}-1\right) \operatorname{deg} u+\max \left\{\operatorname{deg} g_{i, e_{i}}\right. \\
& \left.+\operatorname{deg} g_{j, e_{j}-1}, \operatorname{deg} g_{j, e_{j}}+\operatorname{deg} g_{i, e_{i}-1}\right\}-\operatorname{deg} D_{i j}-1
\end{aligned}
$$

and hence,

$$
\begin{aligned}
\operatorname{deg} u & \leq E+\max \left\{\operatorname{deg} g_{i, e_{i}}+\operatorname{deg} g_{j, e_{j}-1}, \operatorname{deg} g_{j, e_{j}}+\operatorname{deg} g_{i, e_{i}-1}\right\}-1 \\
& \leq E+2 d_{n}-1 .
\end{aligned}
$$

Therefore, for $1 \leq i \leq n, G_{i}(X, u(X))$ is a product of at most $E$ distinct irreducible factors, with degree not exceeding $e_{n}\left(E+2 d_{n}-1\right)+d_{n}$. If $w_{0}, \ldots, w_{E-1}$ are the respective multiplicities of said factors, then up to multiplication by a nonzero constant, the number of possibilities for $G_{i}(X, u(X))$ is at most the number of non-negative integer solutions to the inequality

$$
\sum_{j=0}^{E-1} w_{j} \leq e_{n}\left(E+2 d_{n}-1\right)+d_{n}
$$

which is at most

$$
\left(\begin{array}{c}
e_{n}\left(E+2 d_{n}-1\right)+E+d_{n} \\
E
\end{array}\right)
$$


from Lemma 4.1. For each such possibility, say

$$
G_{i}(X, u(X))=\sum_{j=0}^{d_{i}} \sum_{k=0}^{e_{i}} a_{j k} X^{j} u(X)^{k}=A \prod_{\ell=0}^{E-1}\left(X-\alpha_{\ell}\right)^{b_{\ell}},
$$

if $u$ is monic then $A$ is uniquely determined. Moreover, we have

$$
u(X) \mid A \prod_{\ell=0}^{E-1}\left(X-\alpha_{\ell}\right)^{b_{\ell}}-\sum_{j=0}^{d_{i}} a_{j 0} X^{j}
$$

so there are finitely many possibilities for monic $u$.

For corollary 1.6 , let $F_{i}(X, Y)=f_{i}(X)+Y$, so $G_{i}(X, Y)=f_{i}(X)+Y$ and $H_{i}(X, Y)=1$. Then

$$
R_{i j}(X)=\operatorname{Res}_{Y}\left(g_{i}, G_{j}\right)=f_{j}(X)-f_{i}(X)
$$

has degree at most $d_{n}$, and thus

$$
E=\sum_{1 \leq i<j} \sum_{i<j \leq n} \operatorname{deg} R_{i j} \leq d_{n} \frac{n(n-1)}{2}=C
$$

The result follows from substituting this into (18), noting that $e_{n}=1$ in this case.

\section{Comments}

Considering the case $v<\mu$ (which encompasses the polynomial case) of Theorem 1.1, and additionally Theorem 1.3, it is of interest to obtain upper bounds for the value $e$ when it is finite. That is, bounds for the period of 0 under iteration of a polynomial or rational function $f$. This problem is investigated in various contexts in [5,11,15$17,20]$. Bounds on the values of the values of $\epsilon, \mu$ and $v$ in the rational function case are similarly of interest.

Another problem is to generalise Theorem 1.3 to rational functions. Our approach used for the polynomial case can plausibly be extended to the situation where $v \leq \mu$, mirroring the proof of the relevant case in Theorem 1.1, but applying an appropriate version of the main theorem in [8]. Such an extension, however, is not immediate for the case $\mu<v$.

Also, note that in the case $\mathbb{F}=\mathbb{C}$, Theorem 1.5 may be able to be generalised to several variables, where $F_{i} \in \mathbb{C}\left(X_{1}, \ldots, X_{m}, Y\right)$ and $u \in \mathbb{C}\left[X_{1}, \ldots, X_{m}\right]$, using an appropriate analogue of Mason's theorem (for example [1, Theorem 2]).

Acknowledgements The author is grateful to Alina Ostafe and Igor Shparlinski for their ideas, comments and encouragement. He would also like to thank the referee for a careful reading and valuable suggestions. 
Open Access This article is licensed under a Creative Commons Attribution 4.0 International License, which permits use, sharing, adaptation, distribution and reproduction in any medium or format, as long as you give appropriate credit to the original author(s) and the source, provide a link to the Creative Commons licence, and indicate if changes were made. The images or other third party material in this article are included in the article's Creative Commons licence, unless indicated otherwise in a credit line to the material. If material is not included in the article's Creative Commons licence and your intended use is not permitted by statutory regulation or exceeds the permitted use, you will need to obtain permission directly from the copyright holder. To view a copy of this licence, visit http://creativecommons.org/licenses/by/4.0/.

\section{References}

1. Bayat, M., Teimoori, H.: A new bound for an extension of Mason's theorem for functions of several variables. Arch. Math. 82, 230-239 (2004)

2. Benjamin, A., Bennett, C.: The probability of relatively prime polynomials. Math. Mag. 80, 196-202 (2007)

3. Bombieri, E., Gubler, W.: Heights in Diophantine Geometry, pp. 1-16. Cambridge University Press, Cambridge (2006)

4. Bombieri, E., Masser, D., Zannier, U.: Intersecting a curve with algebraic subgroups of multiplicative groups. Int. Math. Res. Not. 20, 1119-1140 (1999)

5. Canci, J.K.: Finite orbits for rational functions. Indag. Math. 18(2), 203-214 (2007)

6. Carter, S.: Rational function decomposition of polynomials. RHUMJ 13(2), 54-62 (2012)

7. Dubickas, A., Sha, M.: Multiplicative dependence of the translations of algebraic numbers. Revista Matematica Iberoamericana 34, 1789-1808

8. Fuchs, C., Pethö, A.: On composite rational functions having a bounded number of zeros and poles. Proc. Am. Math. Soc. 139, 31-38 (2011)

9. Fuchs, C., Zannier, U.: Composite rational functions expressible with few terms. J. Eur. Math. Soc. 14, 175-208 (2010)

10. Gao, S.: Elements of provable high order in finite fields. Proc. Am. Math. Soc. 127(6), 1615-1623 (1999)

11. Halter-Koch, F., Konec̃ná, P.: Polynomial cycles in finite extension fields. Math. Slovaca 52(5), 531535 (2002)

12. Lambe, T.A.: Bounds on the number of feasible solutions to a knapsack problem. SIAM J. Appl. Math. 26(2), 302-305 (1974)

13. Lidl, R., Niederreiter, H.: Finite Fields. Addison-Wesley, Reading, MA (1983). (Now distributed by Cambridge University Press)

14. Mason, R.C.: Diophantine Equations Over Function Fields, London Mathematical Society Lecture Note Series, vol. 96. Cambridge University Press, Cambridge (1984)

15. Narkiewicz, W.: Polynomial cycles in cubic fields of negative discriminant. Funct. Approx. Comment. Math. 35, 261-269 (2006)

16. Narkiewicz, W., Marszalek, R.: Finite polynomial orbits in quadratic rings. Ramanujan J. 12(1), 91-130 (2006)

17. Narkiewicz, W.: Polynomial cycles in certain rings of rationals. J. Theor. Nombres Bordx. 14(2), 529-552 (2002)

18. Ostafe, A., Sha, M., Shparlinski, I.E., Zannier, U.: On multiplicative dependence of values of rational functions and a generalisation of the Northcott theorem. Michigan Math. J. 68, 385-407 (2019)

19. Popovych, R.: On elements of high order in general finite fields. Algebra Discrete Math. 18(18), 295-300 (2014)

20. Pezda, T.: Polynomial cycles in certain local domains. Acta Arith. 66(1), 11-22 (1994)

21. Silverman, J.H.: Rational fucntions with a polynomial iterate. J. Algebra 180(54), 102-110 (1996)

22. Silverman, J.H.: The S-unit equation over function fields. Proc. Camb. Philos. Soc. 95, 3-4 (1984)

23. Stothers, W.W.: Polynomial identities and Hauptmoduln. Q. J. Math. Oxf. 32(3), 349-370 (1981)

24. Zannier, U.: On the number of terms of a composite polynomial. Acta Arith. 127(2), 157-167 (2007)

Publisher's Note Springer Nature remains neutral with regard to jurisdictional claims in published maps and institutional affiliations. 